\title{
CONSTITUENT LOWER EXTREMITY WORK (CLEW) APPROACH: A NOVEL TOOL TO VISUALIZE JOINT AND SEGMENT WORK
}

\author{
Anahid Ebrahimi, BS ${ }^{\mathrm{a}}$; Saryn R. Goldberg, $\mathrm{PhD}^{\mathrm{b}}$; Jason M. Wilken, PhD, MPT ${ }^{\mathrm{c}}$; Steven J. \\ Stanhope, $P D^{\mathrm{a}, \mathrm{d}, \mathrm{e}, \mathrm{f}}$ \\ ${ }^{a}$ Department of Mechanical Engineering, University of Delaware, Newark, DE, USA \\ ${ }^{\mathrm{b}}$ Department of Engineering, Hofstra University, Hempstead, NY, USA \\ ${ }^{\mathrm{c}}$ Center for the Intrepid, Brooke Army Medical Center, Fort Sam Houston, TX, USA \\ ${ }^{\mathrm{d}}$ Biomechanics and Movement Science Interdisciplinary Program, University of Delaware, \\ Newark, DE, USA \\ ${ }^{\mathrm{e}}$ Department of Kinesiology and Applied Physiology, University of Delaware, Newark, DE, \\ USA \\ ${ }^{\mathrm{f}}$ Department of Biomedical Engineering, University of Delaware, Newark, DE, USA
}

\section{For submission to Gait and Posture as a Short Communication}

WORD COUNT (Abstract): 241 of 250

WORD COUNT (Main text): 1,181 of 1,200

\author{
Corresponding author: \\ Anahid Ebrahimi, BS \\ University of Delaware \\ 540 South College Ave \\ STAR Campus - Room 201 \\ Newark, DE 19713 \\ Phone: (530) 574-7601 \\ E-mail: anahide@,udel.edu
}

\section{ACKNOWLEDGEMENTS}

This material is based upon work supported by the National Science Foundation Graduate Research Fellowship under Grant No. 1247394 and by the University of Delaware College of Health Sciences and Mechanical Engineering departments. Any opinions, findings, and conclusions or recommendations expressed in this material are those of the author(s) and do not necessarily reflect the views of the National Science Foundation. 


\begin{abstract}
Work can reveal the mechanism by which movements occur. However, work is less physically intuitive than more common clinical variables such as joint angles, and are scalar quantities which do not have a direction. Therefore, there is a need for a clearly reported and comprehensively calculated approach to easily visualize and facilitate the interpretation of work variables in a clinical setting. We propose the Constituent Lower ExtremityWork (CLEW) approach, a general methodology to visualize and interpret cyclic tasks performed by the lower limbs. Using 6 degree-of-freedom power calculations, we calculated the relative work of the four lower limb constituents (hip, knee, ankle, and distal foot).In a single pie chart, the CLEW approach details the mechanical cost-of-transport, the percentage of positive and negative work performed in stance phase and swing phase, and the individual contributions of positive and negative work from each constituent. This approach can be used to compare the constituent-level adaptations occurring between limbs of individuals with impairments, or within a limb at different gait intensities. In this paper, we outline how to generate and interpret the CLEW pie chartsin a clinical report. As an example of the utility of the approach, we created a CLEW report using average reference data from eight unimpaired adult subjects walking on a treadmill at 0.8 statures/s $(1.4 \mathrm{~m} / \mathrm{s})$ compared with data from the intact and prosthetic limbs of an individual with a unilateral amputation walking with an above-knee passive prosthesis.
\end{abstract}

KEY WORDS: Gait analysis; Clinical tool; Constituent work; Cost-of-transport 


\section{INTRODUCTION}

Several researchers have used the principles of energetics to explain the compensatory strategies used by individuals with impairments (e.g., [1-3]).Relative joint work, or the comparative amount each joint's work contributed to absolute limb work, can reveal the primary limb “drivers” (positive) and "brakers” (negative) during a movement task like walking. However, work is less physically intuitive than more common clinical variables such as joint angles, partly because it is a scalar quantity which does not have a direction.

Previously, researchers have reported the work generated (positive) and absorbed (negative) by each of the joints using line [1,4] and bar charts [5]. While these graphs can be used to compare joint work across gait intensity and between limbs of the same joint at one intensity, the overlapping lines and error bars can be confusing to interpret. There is a need for a clearly reported approach to visualize and facilitate the interpretation ofwork variables in a clinical setting.

The objective of this paper is to introducethe Constituent Lower ExtremityWork (CLEW) approach, a general methodology to visualize and interpret cyclic tasks performed by the lower limbs. The term "constituents" will be used to refer to the hip, knee, ankle, and distal foot of the limb.The utility of this tool is demonstrated by presenting a report with the relative work of the four lower limb constituentsin both limbs of a sample of healthy, unimpaired individuals and in the prosthetic and intact limbs of an individual with a unilateral amputation walking on a treadmill.

\section{METHODS}

As a representative case study, data were collected from an adult individual (height 1.68 m, mass $79.15 \mathrm{~kg}$ ) walking on an instrumented treadmill (Bertec Corp., Columbus, OH) who 
required use of an above-knee prosthesis due to a congenital proximal femoral focal deficiency. Reflective markers were positioned using a modification of a 6-degree-of-freedom (6-DOF) marker set [6]. A seven-camera motion capture system was used to collect kinematic data (Motion Analysis, Santa Rosa, CA). Motion capture and force data were sampled at $240 \mathrm{~Hz}$ and $1200 \mathrm{~Hz}$ and low-pass filtered at $6 \mathrm{~Hz}$ and $25 \mathrm{~Hz}$, respectively. These data werecompared to data from unimpaired subjects collected as part of a previous study [7] in whicheight healthy adult subjects (height $1.77 \pm 0.08 \mathrm{~m}$, mass71.8 $\pm 15.5 \mathrm{~kg}$ )walked on an instrumented treadmill (Bertec Corp., Columbus, $\mathrm{OH}$ ) while a six-camera motion capture system was used to collect kinematic data (Vicon, Los Angeles, CA) using the same marker set.All subjects walked at a height-scaled speed of 0.8 statures/s (approximately $1.4 \mathrm{~m} / \mathrm{s}$ ) and provided informed consent under an IRB approved protocol.

Methods previously described in the literature [8,9]were used to calculate 6-DOF constituent powersin Visual3D software (C-Motion, Inc. Germantown, MD).A unified deformable segment model was used to characterize the power from the below-knee structures (i.e. combined ankle-foot) of the prosthetic limb during stance phase of the amputee subject [10]. Integrating the positive and negative portions of the constituent power curves over stance and swing phases resulted in the respective constituent work values. The absolute 6-DOFlimb work $\left({ }^{\mathrm{abs}} \mathrm{W}_{\mathrm{limb}}\right)$ was defined as positive limb work summed with the absolute value of negative limb workover the gait cycle (where limb work is defined as summed hip, knee, ankle, and distal foot work). The cost-of-transport is ${ }^{\mathrm{abs}} \mathrm{W}_{\text {limb }}$ Scaled by stride length.Relative work (RW) was the absolute value of each constituent's work divided by the absolute 6-DOFlimb work. Net limb work was the sum of the positive and negative 6-DOFlimb work over both phases. Work valueswere scaled by body mass and averagedforall unimpaired subjects.Distal foot calculations 
werenot relevantduring swing phase.The CLEW approach pie charts were created using the steps depicted in Figure 1.

\section{RESULTS}

Average net 6-DOF limb work, absolute 6-DOFlimb work, stride length, and cost-oftransport are all reported in Table 1 for the left and right limbs of the unimpaired individuals (mean \pm standard deviation)and the individual with amputation (hereafternoted as subject data). Figure 2 depicts a typical clinical CLEW report. Figure 2A summarizes the steps for systematically evaluating a subject's CLEW pie chart and a short interpretation of each variable. Figure 2Bprovides an example of a typical CLEW report with reference data from the unimpaired individuals and a subject's data from the individual with a unilateral amputation.A supplemental Table lists the relative constituent work values for the unimpaired individuals and the subject during stance and swing phases of gait.

\section{DISCUSSION}

The purpose of this study was to introduce the CLEW approach and demonstrate its utility in quantifying relative constituent work in a succinct and visually informative manner.The size of the pie charts, representing the mechanical cost-of-transport, provides a spatial relationship to interpret the total burden of work for the limb. The designation of positive and negative relative constituent work provides a way to readily compare the contribution of work from each constituent during the stance and swing phases of gait, thus identifying the primary “drivers” and "brakers” of the system. 
The CLEW approach pie charts (as in Figure 2B) may beclinically usefulas a way to characterizethe burden of work over an entire stride rather than an instant in time.For example, visual inspection of the size of the pie charts (scaled by cost-of-transport) appears to show greater burden of work (i.e. more absolute 6-DOF limb work) on the intact limb $(1.49 \mathrm{~J} / \mathrm{kg} / \mathrm{m})$ than on the prosthetic limb $(0.67 \mathrm{~J} / \mathrm{kg} / \mathrm{m})$ and compared to the unimpaired limbs $(1.22 \pm 0.15$ $\mathrm{J} / \mathrm{kg} / \mathrm{m}$ on left and $1.19 \pm 0.14 \mathrm{~J} / \mathrm{kg} / \mathrm{m}$ on right). On the prosthetic side, there is almost equal relative limb work (summed positive and negative) from stance(49\%) and swing (51\%), with a majority of the work from the positive hip (24\% in stance, $18 \%$ in swing). A clinician may use this information to test a powered prosthetic device to reduce the burden of work on the intact limb and the hip work on the prosthetic limb, as may be hypothesized from the literature [11].

Future clinical studies will be necessary to determine how a clinical treatment affects the work distribution of the limb. The CLEW approach may be applicable to the upper extremity as well, although this application was not explored here.This was a convenientsample of eight healthy, unimpaired adults, so the values represented here may not be representative of a larger population. A limitation of the 6-DOF approach is that it does not fully capture work due to soft tissue dissipation[12].

The CLEW approach is a comprehensive data visualization tool for representing limb work over a cyclic task, such as overa stride in gait. In a single figure, the CLEW approach details the mechanical cost-of-transport, the percentage of positive and negative work performed in stance phase and swing phase, as well as the individual contributions of positive and negative work from each constituent. Furthermore, the approach can be used to compare the constituentlevel adaptations occurring between limbs of individuals with impairments, or within a limb at different gait intensities. 


\section{ACKNOWLEDGEMENTS}

This material is based upon work supported by the National Science Foundation Graduate Research Fellowship under Grant No. 1247394and by the University of Delaware College of Health Sciences and Mechanical Engineering departments. Any opinions, findings, and conclusions or recommendations expressed in this material are those of the author(s) and do not necessarily reflect the views of the National Science Foundation.

\section{REFERENCES}

[1] L.F. Teixeira-Salmela, S. Nadeau, M.-H. Milot, D. Gravel, L.F. Requião, Effects of cadence on energy generation and absorption at lower extremity joints during gait, Clin. Biomech. 23 (2008) 769-778. doi:10.1016/j.clinbiomech.2008.02.007.

[2] G.S. Sawicki, C.L. Lewis, D.P. Ferris, It pays to have a spring in your step, Exerc. Sport Sci. Rev. 37 (2009) 130-138. doi:10.1097/JES.0b013e31819c2df6.

[3] L.E. Cofré, N. Lythgo, D. Morgan, M.P. Galea, Aging modifies joint power and work when gait speeds are matched, Gait Posture. 33 (2011) 484-489. doi:10.1016/j.gaitpost.2010.12.030.

[4] I.H. Chen, K.N. Kuo, T.P. Andriacchi, The influence of walking speed on mechanical joint power during gait, Gait Posture. 6 (1997) 171-176. doi:10.1016/S09666362(97)00009-X.

[5] A.K. Silverman, N.P. Fey, A. Portillo, J.G. Walden, G. Bosker, R.R. Neptune, Compensatory mechanisms in below-knee amputee gait in response to increasing steadystate walking speeds, Gait Posture. 28 (2008) 602-609. doi:10.1016/j.gaitpost.2008.04.005.

[6] J. Holden, G. Chou, S. Stanhope, Changes in knee joint function over a wide range of walking speeds, Clin. Biomech. 12 (1997) 375-382. doi: 10.1016/S0268-0033(97)00020$\mathrm{X}$.

[7] S.R. Goldberg, S.J. Stanhope, Sensitivity of joint moments to changes in walking speed and body-weight-support are interdependent and vary across joints, J. Biomech. 46 (2013) 1176-1183. doi:10.1016/j.jbiomech.2013.01.001.

[8] F.L. Buczek, T.M. Kepple, K.L. Siegel, S.J. Stanhope, Translational and rotational joint power terms in a six degree-of-freedom model of the normal ankle complex, J. Biomech. 27 (1994) 1447-1457. doi:10.1016/0021-9290(94)90194-5.

[9] K.Z. Takahashi, S.J. Stanhope, Mechanical energy profiles of the combined ankle-foot system in normal gait: insights for prosthetic designs, Gait Posture. 38 (2013) 818-823. doi:10.1016/j.gaitpost.2013.04.002. 
[10] K.Z. Takahashi, T.M. Kepple, S.J. Stanhope, A unified deformable (UD) segment model for quantifying total power of anatomical and prosthetic below-knee structures during stance in gait, J. Biomech. 45 (2012) 2662-2667. doi:10.1016/j.jbiomech.2012.08.017.

[11] S.K. Au, J. Weber, H. Herr, Powered Ankle - Foot Prosthesis Improves Walking Metabolic Economy, IEEE Trans. Robot. 25 (2009) 51-66. doi: 10.1109/TRO.2008.2008747.

[12] K.E. Zelik, K.Z. Takahashi, G.S. Sawicki, Six degree-of-freedom analysis of hip, knee, ankle and foot provides updated understanding of biomechanical work during human walking, J. Exp. Biol. 218 (2015) 876-886. doi:10.1242/jeb.115451. 
Table 1. Net and absolute 6-DOF limb work, stride length, and cost-oftransport for average of a sample $(n=8)$ of unimpaired individuals (mean \pm standard deviation), as well as for an individual subject $(\mathrm{n}=1)$ with a unilateral amputation wearing an above-knee prosthesis.

\begin{tabular}{|l|c|c|c|c|}
\cline { 2 - 5 } \multicolumn{1}{c|}{} & \multicolumn{2}{c|}{ Unimpaired $(\mathbf{n}=8)$} & \multicolumn{2}{c|}{ Subject $(\mathbf{n}=\mathbf{1})$} \\
\cline { 2 - 5 } \multicolumn{1}{c|}{} & Left & Right & Prosthetic & Intact \\
\hline${ }^{\text {net }} \mathrm{W}_{\text {limb }}(\mathrm{J} / \mathrm{kg})$ & $0.05 \pm 0.05$ & $0.11 \pm 0.09$ & 0.02 & -0.06 \\
\hline${ }^{\mathrm{abs}} \mathrm{W}_{\text {limb }}(\mathrm{J} / \mathrm{kg})$ & $1.66 \pm 0.31$ & $1.62 \pm 0.28$ & 0.90 & 1.77 \\
\hline Stride length $(\mathrm{m})$ & $1.36 \pm 0.14$ & $1.36 \pm 0.14$ & 1.34 & 1.19 \\
\hline Cost-of-transport $(\mathrm{J} / \mathrm{kg} / \mathrm{m})$ & $1.22 \pm 0.15$ & $1.19 \pm 0.14$ & 0.67 & 1.49 \\
\hline
\end{tabular}




\section{Figure Legends}

Figure 1. Steps for creating the CLEW approach pie charts for gait. (A) First, the area of the pie chart is scaled to the cost-of-transport (COT). Here $1 \mathrm{~J} / \mathrm{kg} / \mathrm{m}$ is a circle with area of $25.0 \mathrm{~cm}^{2}$. The central reference line is defined from the center of the circle to the top. The COT is displayed in the center of the pie chart in a white circle. (B) Second, the relative constituent work contributions to absolute 6-DOF limb work during swing phase are grouped together and designated visually by a partial pie slice. From the central reference line, positive work will be to the left side of the circle (dark), while negative work is to the right (light). (C) Third, each relative constituent work is designated its portion of the pie, in the order of hip, knee, ankle, and distal foot (or ankle-foot) for swing, then stance, beginning from the central reference line. The net work line at the bottom of the circle separates the positive from the negative work in stance phase.

Figure 2. (A) General approach for evaluating data from the CLEW report. This guide can be used to assess the CLEW pie charts systematically. Note, if unimpaired reference data is used, left and right limbs may be grouped together when appropriate. (B) Example CLEW report with average data from unimpaired individuals $(n=8)$ walking at 0.8 statures/s serving as reference data. Subject data are from an individual with a unilateral amputation $(n=1)$ walking at 0.8 statures/s. The unified deformable segment model [20] was used to characterize the work from the below-knee structures of the prosthetic limb during stance phase, noted here as Ankle-Foot (AF). 

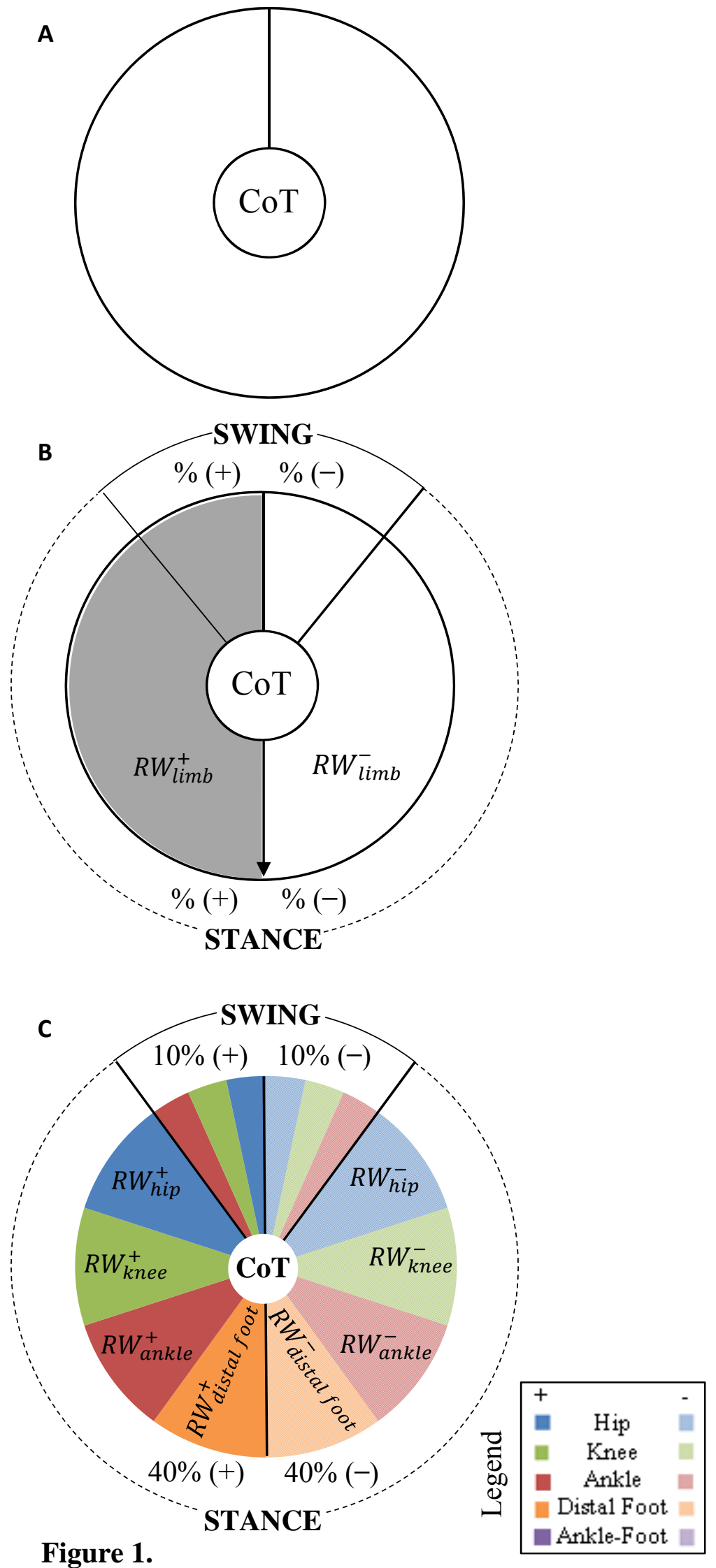

Figure 1. 


\section{CLEW Report: General Approach}

Task: (e.g. walking, sit-to-stand)

Phase: (e.g. stance, swing)

Scale: (e.g. cost-of-transport scaled to area of pie)

\section{REFERENCE DATA}

Examples of Reference data (left and/or right):

- Average data from individuals without impairments

- Average data from individuals with the same impairment as the Subject

- A prior assessment from the same Subject

Key for Constituents:

H: Hip, K: Knee, A: Ankle, F: Distal Foot, AF: Ankle-Foot*

Relative work not labeled for pie slices $<2 \%$

SUBJECT LIMBS

1a. The area (size) of the pie chart represents total cost-oftransport (CoT). Compare total CoT between limbs.

1b. Compare the Subject's total CoT to the Reference.

2a. Compare the portion of positive $(+)$ and negative (-) relative work $(\mathrm{RW})$ within the same limb (within or between stance and swing phase).

2b. Compare the portion of summed $(+)$ and $(-)$ relative work between limbs and relative to the reference data.

3. Compare positive and negative RW of constituents in each phase to respective constituents of opposite limb and to the Reference data.

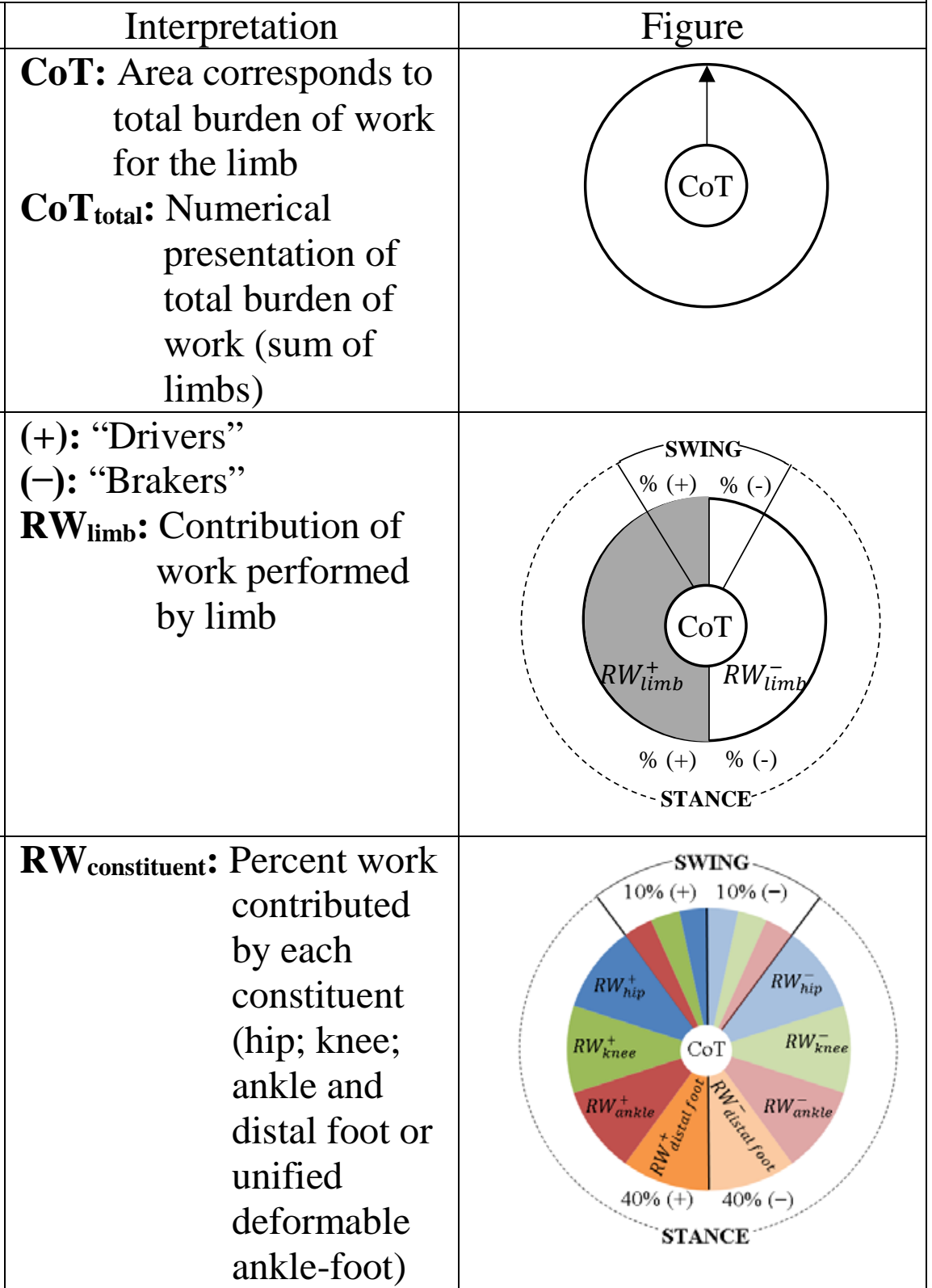


CLEW Report: Example

Task: Walking

Phase: Stance, Swing

Scale: $1 \mathrm{~J} / \mathrm{kg} / \mathrm{m}: 25 \mathrm{~cm}^{2}$
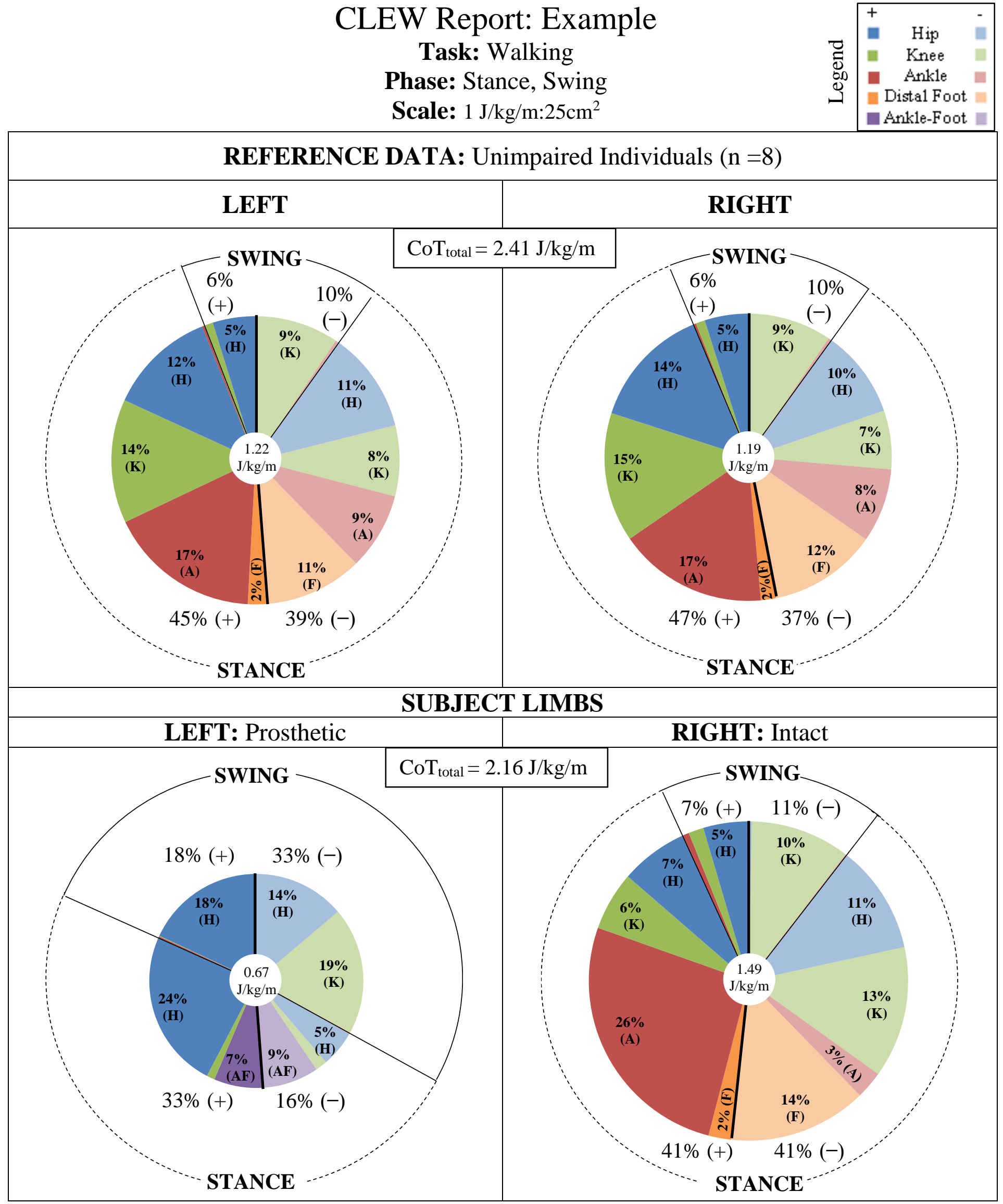

Key: H: Hip; K: Knee; A: Ankle; F: Distal Foot; AF: Unified Deformable Ankle-Foot* Relative work not labeled for pie slices $<2 \%$; *See Takahashi et al., 2012.

Figure 2B. 


\section{CLEW Report: General Approach}

Task: (e.g. walking, sit-to-stand)

Phase: (e.g. stance, swing)

Scale: (e.g. cost-of-transport scaled to area of pie)

\section{REFERENCE DATA}

Examples of Reference data (left and/or right):

- Average data from individuals without impairments

- Average data from individuals with the same impairment as the Subject

- A prior assessment from the same Subject

Key for Constituents:

H: Hip, K: Knee, A: Ankle, F: Distal Foot, AF: Ankle-Foot*

Relative work not labeled for pie slices $<2 \%$

SUBJECT LIMBS

1a. The area (size) of the pie chart represents total cost-oftransport (CoT). Compare total CoT between limbs.

1b. Compare the Subject's total CoT to the Reference.

2a. Compare the portion of positive $(+)$ and negative (-) relative work $(\mathrm{RW})$ within the same limb (within or between stance and swing phase).

2b. Compare the portion of summed $(+)$ and $(-)$ relative work between limbs and relative to the reference data.

3. Compare positive and negative RW of constituents in each phase to respective constituents of opposite limb and to the Reference data.

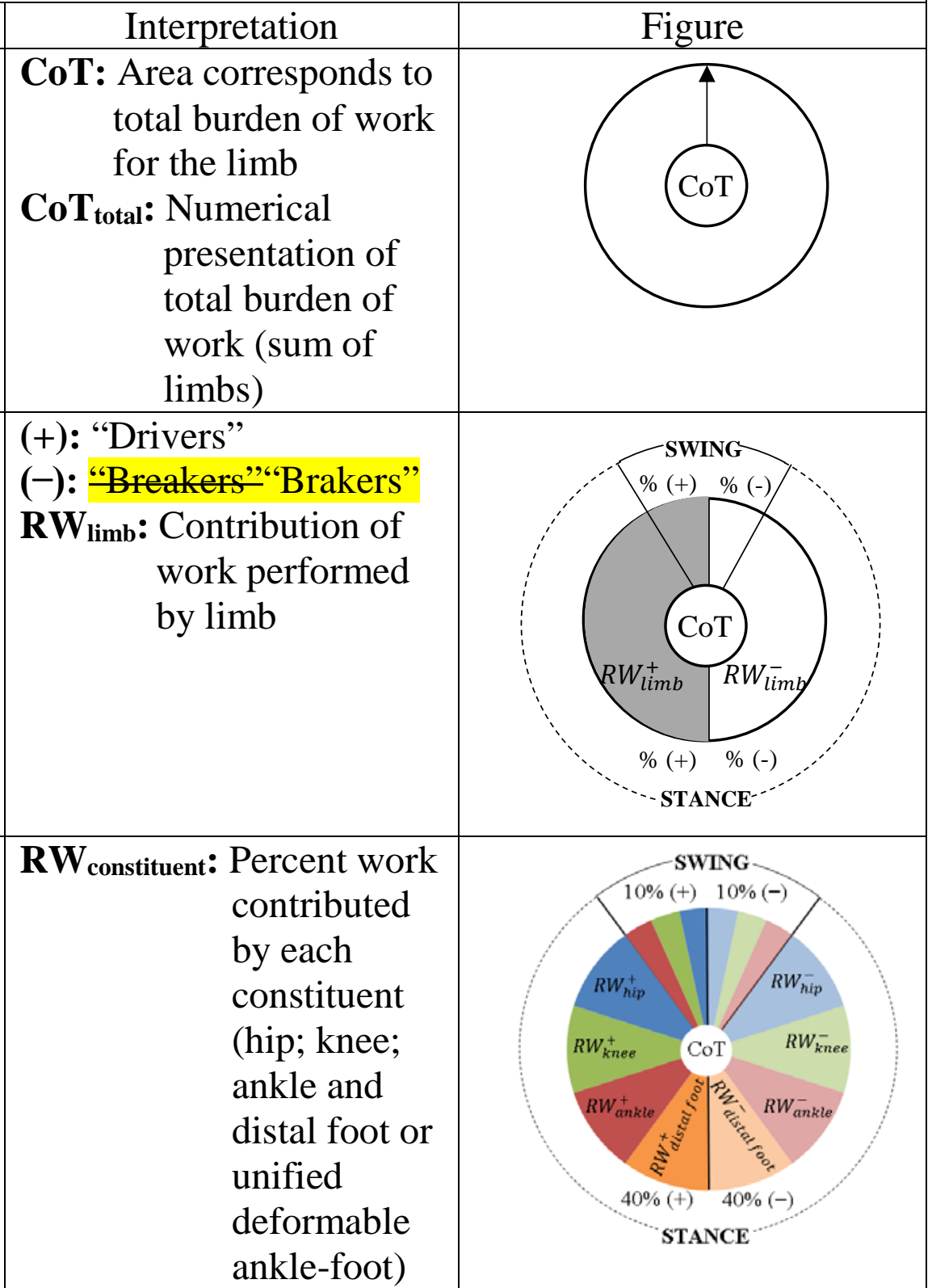


CLEW Report: Example

Task: Walking

Phase: Stance, Swing

Scale: $1 \mathrm{~J} / \mathrm{kg} / \mathrm{m}: 25 \mathrm{~cm}^{2}$
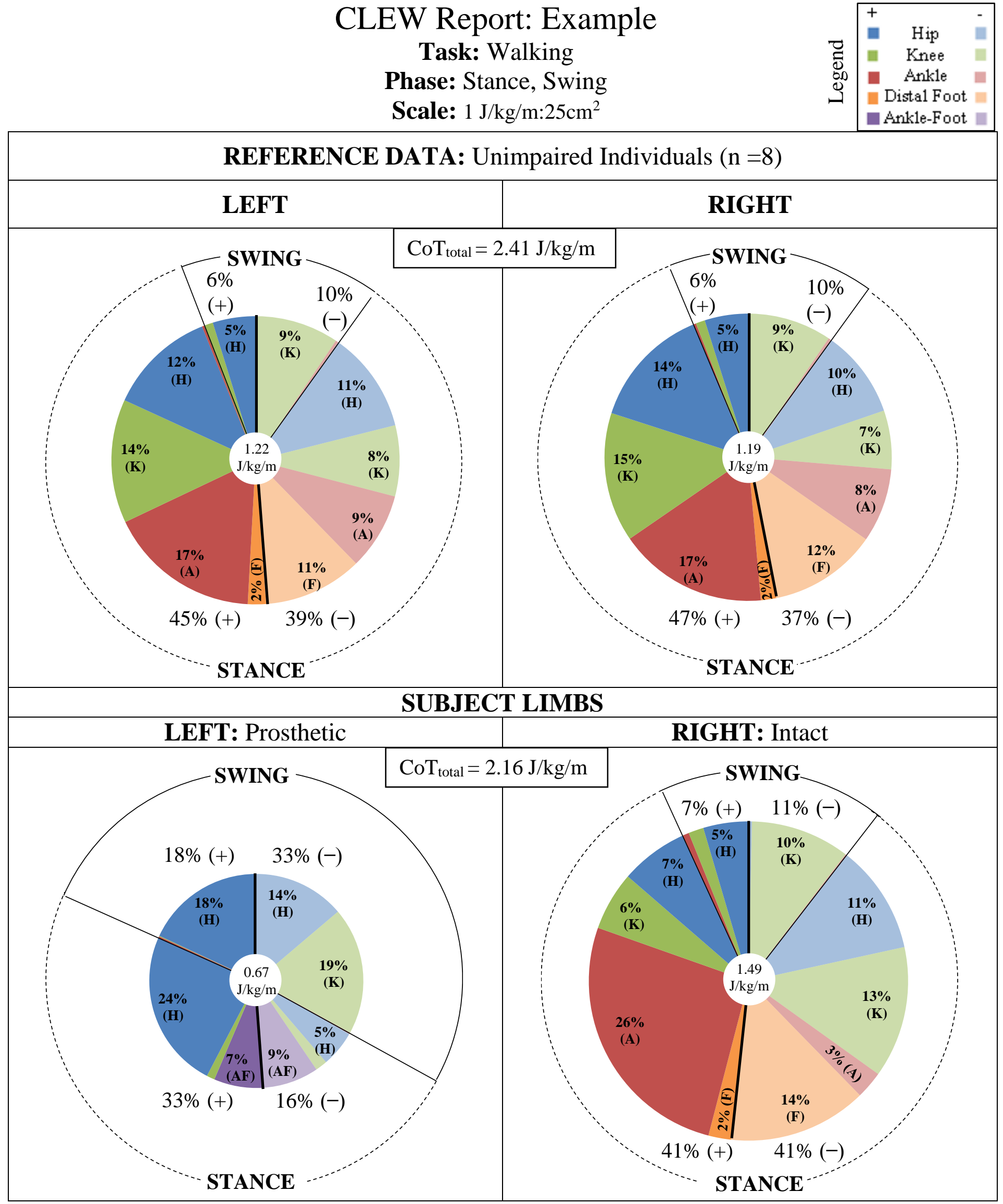

Key: H: Hip; K: Knee; A: Ankle; F: Distal Foot; AF: Unified Deformable Ankle-Foot* Relative work not labeled for pie slices $<2 \%$; *See Takahashi et al., 2012.

Figure 2B. 